\title{
Mental imagery abilities in adolescents with spastic diplegic cerebral palsy
}

\author{
Yanick Courbois $^{1}$, Yann Coello \\ Laboratoire URECA, Université Charles de Gaulle, France \\ ISABELLE BOUCHART
}

Foyer H. Grafteaux, Villeneuve d'Ascq, France

\begin{abstract}
Four visual imagery tasks were presented to three groups of adolescents with or without spastic diplegic cerebral palsy. The first group was composed of six adolescents with cerebral palsy who had associated visual-perceptual deficits $(C P-P D)$, the second group was composed of five adolescents with cerebral palsy and no associated visual-perceptual deficits (CP), and the third group comprised ten control participants. Results revealed a marked slowdown of visual imagery processing in the CP-PD group only. They suggested that cerebral palsy is not systematically associated with visual imagery deficits, and that the presence or absence of such deficits depends on the existence of associated perceptual deficits.
\end{abstract}

\section{Introduction}

Cerebral palsy (CP) is a nonprogressive developmental disorder, impairing control of movement and posture, that results from a brain disorder suffered either before or at birth or during infancy (i.e., foetal diseases, severe jaundice of the new-born, premature birth, perinatal asphyxia). In the spastic diplegic subtype of cerebral palsy, brain injury produces characteristic motor deficits that primarily affect normal functioning of the legs. Spasticity refers to increased tension in muscles, which are stiff and often involuntarily contracted, as a consequence of impairment of temporal control of muscle activity. It has also been established that a large number of persons with cerebral palsy have associated visual deficits such as vision field contraction or oculomotor problems (Black, 1980), leading to visual-perceptual disorders (Breakley, Wilson, \& Wilson, 1974). The latter may, in turn, account for difficulties that some persons with cerebral palsy encounter in spatial problem solving or learning tasks (e.g., see Foreman, Orencas, Nicholas, Morton, \& Gell, 1989; Mazeau, 1989). One may assume that visual-perceptual

\footnotetext{
${ }^{1}$ Address for correspondence: Yanick Courbois, Laboraratoire URECA, Université Charles de Gaulle, Lille3, BP 149, 59653 Villeneuve d'Ascq Cedex, France. E-mail: courbois@univ-lille3.fr
} 
disorders also affect visual imagery abilities in these persons and the present study was designed to address this issue.

There is a large body of evidence showing that visual perception and visual mental imagery share common processes and neural substrates. This accounts for the functional and structural analogies between visual perception and visual imagery that have frequently been reported in the literature (see Denis, 1991; Finke, 1989; Kosslyn, 1994; Richardson, 1999). Given these close connections, one can expect a theory of visual perception to be also a theory of mental imagery. Among the authors who have tackled this issue, Kosslyn has developed a theory which accounts for a broad range of data obtained in visual perception and mental imagery (Kosslyn, 1994). According to this theory, a mental image is a transient internal representation, which depicts an object, a scene or an event. This representation is generated by activating information in a structure called the visual buffer, which acts as a temporary medium for this purpose. The visual buffer can be activated by information coming from the visual system or from visual representations stored in long-term memory. It is modelled as an Euclidean space (see Kosslyn, 1980) and a visual mental image consists of a pattern of activation in this space. Functional magnetic resonance imaging (fMRI) studies have suggested that the visual buffer is localised in a set of topographically mapped visual areas in the cortex. These primary visual areas, which are typically involved in visual perception, were also found to be activated while participants generated mental images (retinotoptic area of the visual cortex) (Kosslyn et al., 1993; Kosslyn, Thompson, Kim, \& Alpert, 1995). Furthermore, mental imagery is not a unitary and undifferentiated phenomenon but rather is accomplished by a set of subabilities, or subsystems, which operate independently. These processing subsystems work together to upgrade, maintain, transform or analyse information represented in the visual buffer. Very briefly, some of these subsystems are involved in the processing of object properties (shape, colour and texture), others are concerned with spatial properties (location, size, orientation), associative memory or orientation of attention (see Kosslyn, 1994).

It is likely that persons with cerebral palsy who have perceptual deficits should also have low imagery abilities since visual imagery and visual perception share common mechanisms. In order to test this hypothesis, we constituted two groups of adolescents with cerebral palsy on the basis of medical and clinical assessments. Participants of the first group showed strong perceptual deficits whereas those of the second group did not show such deficits. The performances of both groups in four imagery tasks were also compared to those of a control group consisting of 12-year-old children without deficits. The tasks we used were administered on a microcomputer. They were designed to probe four natural functions of visual imagery: (a) generation of an image in the visual buffer from its representation in long term memory (LTM); (b) temporary maintenance of the image in the buffer; (c) inspection of the different parts of the image (image scanning); and $(\mathrm{d})$ mental rotation.

The image generation task required participants to form an image of a block letter within a $4 \times 5$ grid that contained a dot in one cell. The participants were asked to decide whether the letter would have covered the dot if it were actually present. This task was based on the one developed by Kosslyn and his collaborators (see Kosslyn, 1994, p. 299). Kosslyn, Cave, Provost, and Von Gierke (1988) showed that participants required more time to generate visual images of more complex letters (e.g., "G" compared to "L"; complexity refers to the number of segments of the letter) and revealed that generation took place sequentially (i.e., segment by segment). The image maintenance task was 
adapted from the one used by Kosslyn et al. with children and adults (Kosslyn, Margolis, Barrett, Goldknopf, \& Daly, 1990). In this task, the participants first studied a pattern within a grid. Once the pattern was memorised, it was removed and two dots appeared within the empty grid. The participants indicated whether both dots fell in cells that had been filled by the pattern. The task had two conditions, which differed in the complexity of the stimulus and retention duration. The image scanning task was a variant of the one developed by Finke and Pinker (1982). Participants were shown three circles displayed on the periphery of the screen and had to memorise their location. The circles were removed, an arrow appeared very briefly at the centre of the screen, and then the entire display disappeared. Participants had to decide whether the arrow was pointing towards a circle or not. We varied the distance from the arrow to the circle, since it has repeatedly been found that the duration of scanning is proportional to the distance covered on the image (see Denis \& Kosslyn, 1999). We assessed mental rotation abilities using two tasks that required the participants to decide whether two shapes were identical, regardless of their orientation (see Shepard \& Metzler, 1971). The tasks differed according to the ease of encoding of the stimulus; i.e., the vertical axis was salient for one stimulus $(\mathrm{S}+)$ but not for the other $(\mathrm{S}-)$. Courbois $(2000)$ found that mental rotation of $\mathrm{S}-$ stimuli was more difficult than mental rotation of $\mathrm{S}+$ stimulus and that there were strong developmental differences in the ability to mentally rotate $\mathrm{S}-$ stimuli.

We expected the performances in these four tasks to be poorer for the group with perceptual deficits associated with cerebral palsy than for the two other groups. Comparisons between these latter groups would also allow us to unravel whether cerebral palsy is systematically associated with difficulties in visual imagery.

\section{Method}

\section{Participants}

Eleven adolescents with spastic diplegic cerebral palsy were selected on the basis of medical and psychometric information. They were aged from 12 years 5 months to 14 years 9 months and attended mainstream high school. Clinical reading of records revealed that six of them had strong deficits in visual perception ( 5 males and 1 female). These participants had low scores in psychometric tasks such as the Frostig developmental test of visual perception and the Primary Mental Abilities Battery, and also had difficulties in copying geometrical figures (e.g., a cube). They constituted the CP-PD group (cerebral palsy with perceptual deficits, mean age: $12.8 ; S D=0.61$ ). The other five adolescents had no known deficits in visual perception (4 males, 1 female). They constituted the CP group (mean age: $13.8 ; S D=0.62$ ). Ten adolescents ( 6 males and 4 females) aged from 10 years 2 months to 13 years 5 months constituted the control group (CTRL, mean age: $12 ; S D=1$ ). All participants had normal or corrected to normal vision. None of them had trouble using the response keys on the keyboard.

\section{General procedure}

All the participants were tested individually. The administration of the four tasks was spread over two sessions for the participants with cerebral palsy and one session for the control group. The order of presentation of the tasks was counterbalanced. 
The tasks were administered using a microcomputer. Participants were instructed that following the presentation of each stimulus they would have to give, as fast as possible, the correct answer "yes" or "no" by pressing the red ("V") or blue ("N") key on the keyboard. Responses and response times were recorded automatically by the computer. Duration of item presentation was not restricted and interstimulus interval was 2 seconds.

\section{The image generation task}

Material. Four uppercase letters (H, F, S, and G) and the corresponding lowercase letters were used as stimuli. Two of these uppercase letters, both of which contained three segments, were considered to be relatively simple $(\mathrm{H}, \mathrm{F})$. The two others contained five segments and were considered as being more complex $(\mathrm{S}, \mathrm{G})$. In the test phase, the stimulus was centred on the screen and the grid occupied a visual angle of approximately $5.5^{\circ} \times 7.5^{\circ}$ at a viewing distance of $50 \mathrm{~cm}$. Cards depicting the uppercase letters in the grid and the lowercase letter beneath it were used for familiarisation. Transparencies representing the grid without letters were also used in the familiarisation phase.

Procedure. Before the experiment proper began, participants were shown the four cards representing uppercase letters in the grid and were given practice in reproducing these letters on the transparencies. All were able to reproduce the letters in the grid. Then, they were given four familiarisation trials that were of the same format as the imagery condition trials. These trials consisted of the following sequence: A centred lowercase letter (indicating which block letter to visualise) was displayed for $500 \mathrm{~ms}$, $1000 \mathrm{~ms}$ later the grid with a dot somewhere within appeared for $200 \mathrm{~ms}$. Participants were asked to decide whether the dot would fall on the uppercase version of the letter if it was present. If so, they had to press the "yes" key; if not, the "no" key. The uppercase letter appeared in the grid after the participants had given their response (visual feedback). The generation task was then administered. It consisted of 24 items, 6 per letter. In half of the items, the correct response was "yes"; in the other half, it was "no". No visual feedback was provided following the participants' response. Finally, a perceptual control condition was administered. This condition was exactly analogous to the imagery condition except that the block letter was drawn in the grid. The participants simply had to decide whether the dot was on the letter or not. This condition was also made up of 24 items. It was always administered after the imagery condition to avoid any learning of the dot locations before the generation task.

\section{The image maintenance task}

Material. A $4 \times 5$ grid was used as a stimulus in the "light load" condition, and a $5 \times 7$ grid in the "heavy load" condition (subtended approximately $5.5^{\circ} \times 7.5^{\circ}$ of visual angle). On each trial, $20 \%$ of the cells were randomly filled by the program to form a homogeneous pattern (each filled cell had at least one side or angle connected to another cell). The pattern was removed when the participants pressed the spacebar. Half a second later in the "light load" condition, or three seconds later in the "heavy load" condition, two circles appeared in different cells of the grid. In half of the trials, both circles fell in a cell previously occupied by the pattern, and in the other half, one of the circles fell in a previously empty cell.

Procedure. The task started with a familiarisation phase including four trials. In this 
phase, a pattern of four cells was generated randomly in a $4 \times 5$ grid. The participants studied the pattern and pressed the space bar when they had memorised it.

Half a second after the removal of the image from the grid, two circles appeared on the screen and participants were asked to state whether the two dots fell in cells previously occupied by the viewed pattern. After the participants had given their response, auditory feedback was provided for correct responses and the pattern reappeared. The test phase was composed of two blocks of ten trials (one for the "light load" condition and the other for the "heavy load" condition). On half of the trials, the correct response was "yes" and on the other half "no". Trials were presented in a random order, with the constraint that no more than three successive items could have the same response. The "light load" condition was administered before the "heavy load" one.

\section{The image scanning task}

Material. Three circles (diameter: $4 \mathrm{~cm}$ ) were displayed on the screen, with each circle on a different side of the screen. A $1.5 \mathrm{~cm}$ arrow was presented in one of eight different orientations, from the upright position to $315^{\circ}$, varying with a $45^{\circ}$ increment. The arrow was positioned at three distances from a target circle (near, medium or far). On half of the trials for each distance it pointed to the dot, and on the other half it did not.

Procedure. The task comprised an imagery condition and a perceptual condition. In the imagery condition, three circles were displayed on the screen and the participants were asked to memorise their locations. When participants pressed the space bar, the circles were removed and, $50 \mathrm{~ms}$ latter, an arrow appeared for $200 \mathrm{~ms}$. Participants pressed the "yes" key if the arrow pointed to the circle and the "no" key if not. In the perceptual control condition, the circles did not disappear before the arrow was shown. This condition was always administered after the imagery condition. Each condition consisted of 24 items ( 8 per inspection distance: 4 "yes" and 4 "no") and was preceded by a familiarisation phase which was identical to the test situation except that responses received feedback.

\section{The mental rotation tasks}

Material. The S+ and S- stimuli had the same "structure". They were composed of four arms that were identical in both stimuli. The only difference between the two kinds of stimuli was that the upper arm was more salient in the $\mathrm{S}+$ stimulus than in the $\mathrm{S}-$ stimulus (see Figure 1). Transparencies with reproductions of the stimuli were used in the familiarisation phase. In the mental rotation task, pairs of stimuli were presented side by side. The left stimuli were always upright whereas the right stimuli were presented at $0^{\circ}$, $45^{\circ}, 90^{\circ}, 135^{\circ}$ or $180^{\circ}$. The inclined stimulus was either identical to the other one, or was its mirror image.

Procedure. The $\mathrm{S}-$ condition was always administered before the $\mathrm{S}+$ condition. Each condition began with a familiarisation phase. Transparencies with reproductions of the stimuli were used in this phase, which was composed of eight trials. Two transparencies showing the stimuli in a vertical position were presented to the children. The stimuli were either identical or a mirror image and the participants were asked to say whether they were the same. Once the response had been obtained, the experimenter superimposed the two transparencies to allow the participants to check their answer. The next phase was a criterion test that was administered with the computer. Participants saw pairs of stimuli 

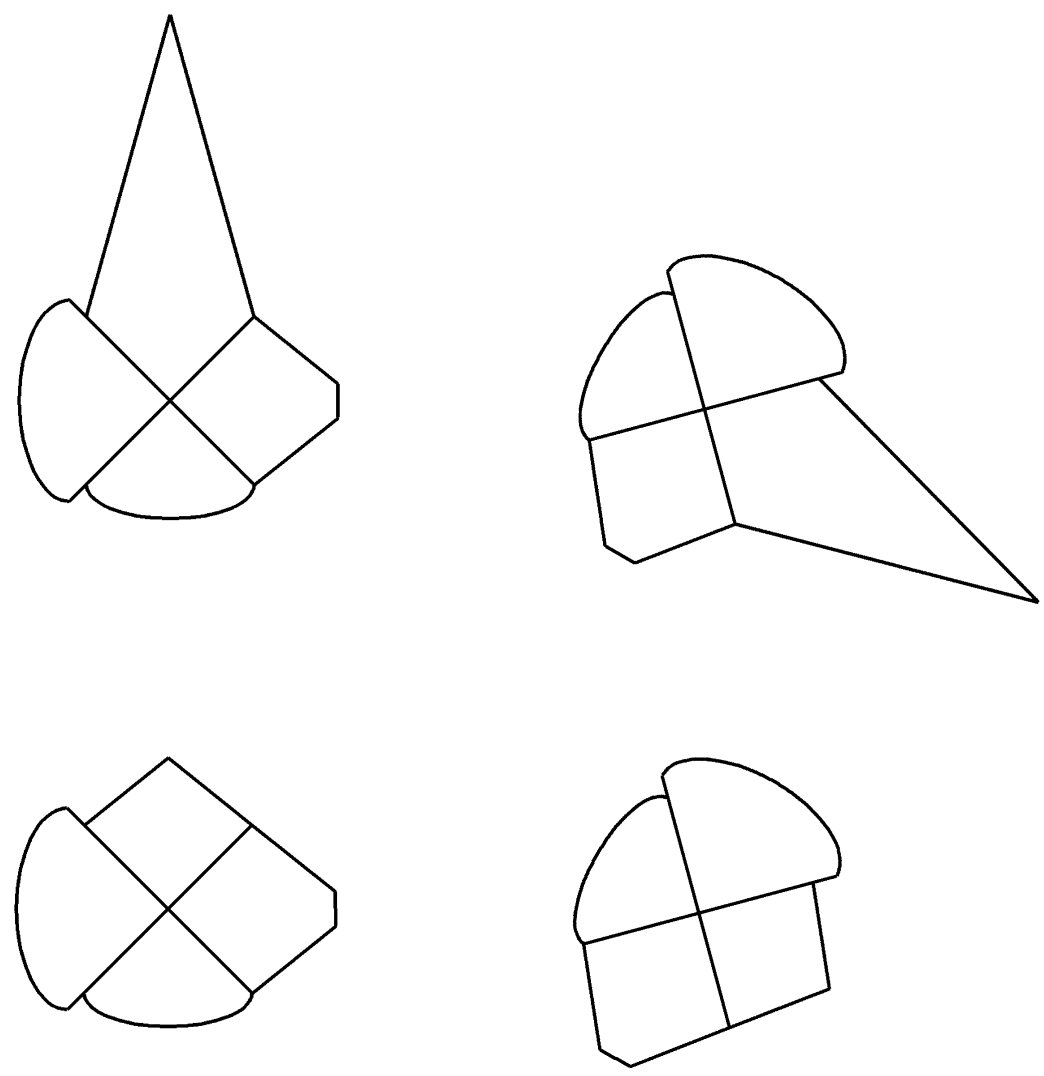

Figure 1.

Mental rotation task: Example of trial using the stimuli with a salient axis (upper panel, orientation: $120^{\circ}$, response "same"), and the corresponding trial using stimuli with no salient axis (lower panel).

presented vertically and had to judge whether they were identical. There was no feedback and participants were selected for the next phases if they responded correctly on 10 out of 12 items.

Participants who passed the criterion test were given a second familiarisation phase and the experimental test. For this last familiarisation phase, pairs of transparencies were presented to the participants. The left-hand stimulus was vertical. The right-hand stimulus was inclined and was either identical to the left-hand one or its mirror image. The participants' task was to determine whether the two stimuli were identical. After each response, the experimenter manually rotated the right-handed stimulus to upright in order to allow the participants to check their response. This familiarisation phase was composed of five trials (one per orientation). The experimental test was administered using the computer. It was identical to the preceding familiarisation phase but no feedback was provided after the response. The task consisted of 30 items ( 6 per orientation). On half of the items the correct response was "yes", for the other half, "no". The items were administered in a random order with the following restrictions: the 
same orientation could not be presented twice in a row, the same response could not be given more than three times in succession.

\section{Results}

The outliers were converted before carrying out the statistical analyses on response times. It was assumed that these values were linked to fluctuations in attention. As in Kosslyn et al. (1990), an outlier was defined as equal to or exceeding twice the mean response times for the participant in this condition. Outliers were replaced by the mean of the remaining times in the cell but there was only one replacement per condition made. This rule was applied for the entire set of tasks ( $1 \%$ of response times were outliers).

\section{Image generation task}

Response times. An analysis of variance was conducted on response time with group, condition and complexity as independent variables. Response time varied significantly as a function of groupla, $\mathrm{F}(2,18)=11.93, p<0.01$, (see Figure 2). Response times were shorter for the CTRL and CP groups compared to the CP-PD group, Duncan test, $p<0.05$. Complexity increased response times, $\mathrm{F}(1,18)=16.48, p<0.01$, but this effect was limited to the imagery condition, as shown by the significant interaction between
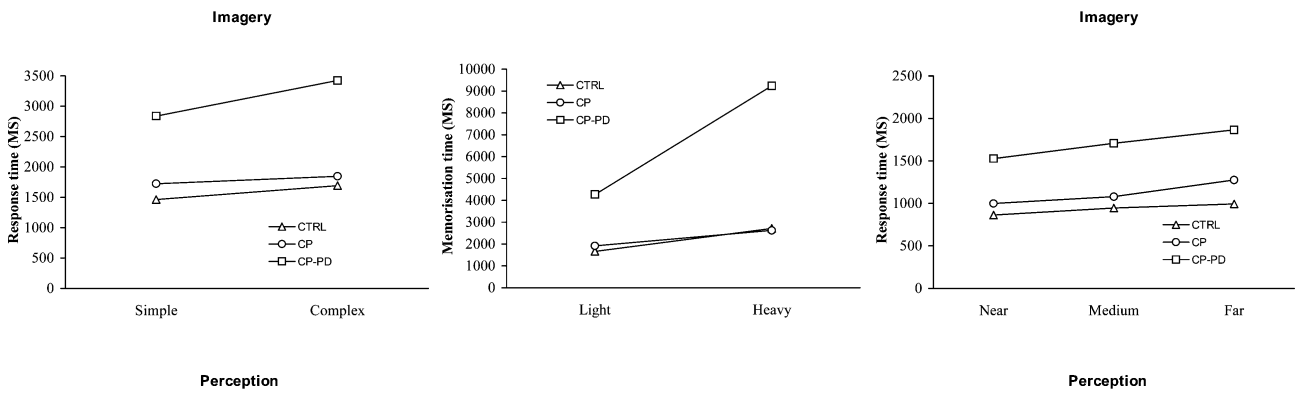

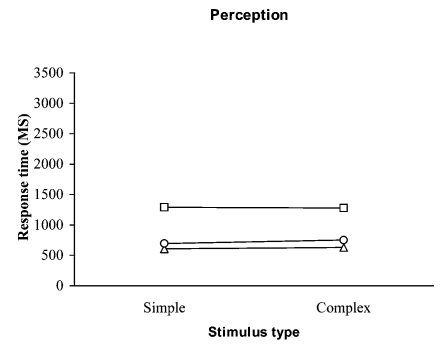

IMAGE GENERATION

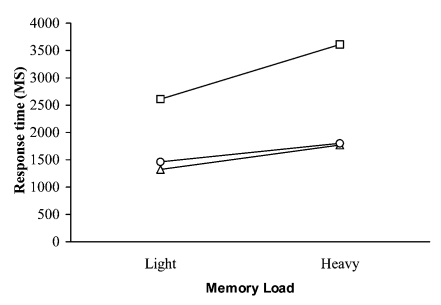

IMAGE MAINTENANCE

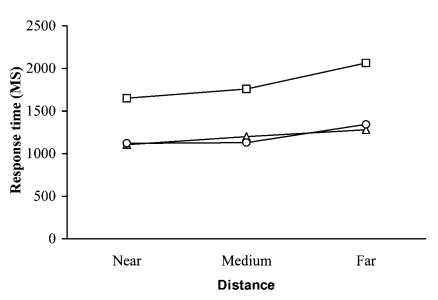

IMAGE SCANNING

Figure 2.

Image generation: Mean response times (in milliseconds) as a function of group, condition (imagery, perception), and stimulus type (simple, complex). Image maintenance: Mean memorisation times in milliseconds (upper panel), and response times (lower panel) as a function of group and memory load (light, heavy). Image scanning: Mean response times (in milliseconds) as a function of group, condition (imagery, perception), and distance. 
stimulus complexity and condition, $\mathrm{F}(1,18)=12.22, p<0.01$. The interaction between group and condition was also significant, $\mathrm{F}(2,18)=8.16, p<0.01$. The differences between the CP-PD group and the two other groups increased in the imagery condition. Finally, a three-way interaction between group, complexity, and condition, $\mathrm{F}(2$, $18)=3.45, p<0.05$, can be accounted for by the stronger effect of complexity in imagery condition for the CP-PD group as compared to the two other groups (see Figure 2).

Error rates. An analysis of variance was conducted on the error rates on the same independent variables as described above. The participants made more errors in the imagery condition than in the perception condition, $11.9 \%$ versus $2.3 \%, F(1,18)=18.6$, $p<0.01$. They also made more errors for the complex than for the simple letters, $8.4 \%$ versus $5.8 \%, \mathrm{~F}(1,18)=3.77, p<0.05$. There was no significant effect of group and no significant interaction ( $p>0.15$ in all cases).

\section{Image maintenance task}

Memorisation times. An analysis of variance was conducted on the memorisation times, using group and load as independent variables. Memorisation times varied significantly as a function of group, $\mathrm{F}(2,18)=13.05, p<0.01$. They were longer for the CP-PD group compared to the CP or the CTRL groups, Duncan test, $p<0.05$. Memorisation times were also longer in the heavy load condition, $\mathrm{F}(1,18)=15.31, p<0.01$. The interaction between load and group was significant, $\mathrm{F}(2,18)=5.56, p<0.05$, due to the fact that the differences between the CP-PD group and the CP and CTRL groups increased in the heavy load condition (see Figure 2).

Response times. An analysis of variance was conducted on the response times using group and load as independent variables. Response times varied significantly as a function of group, $\mathrm{F}(2,18)=9.82, p<0.01$. They were shorter for the control and $\mathrm{CP}$ groups compared to the CP-PD group, Duncan test, $p<0.05$. They were also significantly longer for heavy load items, $\mathrm{F}(1,18)=12.72, p<0.01$ (see Figure 2). The interaction between group and load was not significant, $\mathrm{F}(2,18)=1.43$, n.s.

Error rates. The analysis of variance conducted on the error rates revealed that performance varied significantly across groups, $\mathrm{F}(2,18)=8.61, p<0.01$. The CP-PF group had higher error rates than the control group, $20.2 \%$ versus $7.5 \%$, Duncan test, $p<0.05$ (error rates for the CP group: 14\%). The increase in load resulted in a sharp increase in error rates, $7.8 \%$ versus $20 \%, \mathrm{~F}(1,18)=12.36, p<0.01$, and there was a trend for this effect to be more pronounced in CP and CP-PD groups (interaction between group and load: $\mathrm{F}[2,18]=2.77, p<0.08)$.

\section{Image scanning task}

Response times. An analysis of variance was conducted on the response times with group, distance, and condition as independent variables. As shown in Figure 2, response times increased significantly with distance, $\mathrm{F}(2,36)=43.56, p<0.01$ (linear trend: $\mathrm{F}[1,36]=$ $84.84, p<0.01)$ and were higher in the perceptual condition, $\mathrm{F}(1,18)=4.93, p<0.05$. There were significant differences in response times as a function of group, $\mathrm{F}(2,18)=$ $8.58, p<0.01$. Response times were higher for the CP-PD group compared to the CP or the control groups, Duncan test, $\mathrm{p}<.05$. The interaction between group and distance was also significant, $\mathrm{F}(4,36)=3.97, p<0.01$. Specific analysis revealed that the distance effect 
was stronger for the CP-PD group compared to the control group, $F(2,28)=9.98$, $p<0.01$ (see Figure 2).

Error rates. An analysis of variance conducted on the same independent variables as above was performed on error rates. The only significant effect was an effect of condition, $\mathrm{F}(1,18)=18.07, p<0.01$. Performance was lower in the imagery condition than in the perception condition $(12.2 \%$ versus $5.5 \%)$.

\section{Mental rotation tasks}

Response times. All participants met the criterion. An analysis of variance with group, orientation and stimulus as independent variables was calculated on response times. This analysis confirmed the influence of the group factor, as shown in Figure 3 , $\mathrm{F}(2,18)=29.03, p<0.01$. The CP-PD group had longer response times than the two

\section{S+}

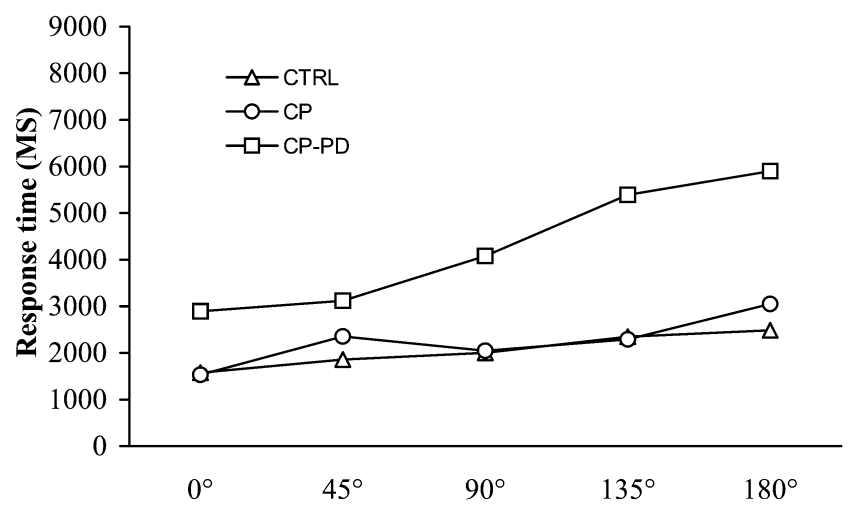

S-

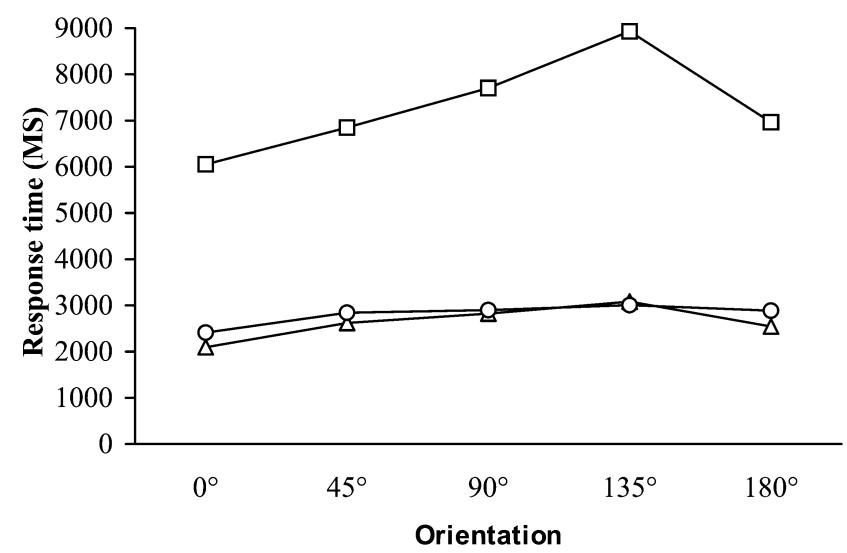

Figure 3.

Mental rotation: Mean response times (in milliseconds) as a function of group, stimulus $(S+, S-)$, and orientation. 
other groups, Duncan test, $p<0.05$. Response times increased with greater angular disparity, $\mathrm{F}(4,72)=19.06, p<0.01$ (linear trend: $\mathrm{F}[1,64]=65.06, p<0.01$ ) and were significantly longer with $\mathrm{S}-$ stimulus, $\mathrm{F}(1,18)=45.91, p<0.01$. The interaction between group and stimulus was significant, $\mathrm{F}(2,18)=15.42, p<0.01$, due to the fact that the differences between the CP-PD group and the two other groups increased with the $\mathrm{S}-$ stimulus. The increase in response times as a function of the orientation was also higher for the CP-PD group (interaction between group and orientation: $F[8,72]=4.15$, $p<0.01)$. A significant interaction between stimulus and orientation, $\mathrm{F}(4,72)=6.70$, $p<0.01$, could be accounted for by a drop in response times between the orientations $135^{\circ}$ and $180^{\circ}$ for the $\mathrm{S}-$ stimulus (this interaction was not significant when data from the orientation $180^{\circ}$ were discarded).

Error rates. The two significant effects in the analysis of variance conducted on error rates were the orientation effect, $\mathrm{F}(4,72)=7.47, p<0.01$, and the stimulus effect, $\mathrm{F}(1,18)=20.95, p<0.01$. Error rates were higher with $\mathrm{S}-$ stimulus $(24.5 \%$ versus $8.6 \%)$ and increased with orientation $\left(0^{\circ}=9.4 \% ; 45^{\circ}=12.9 \% ; \quad 90^{\circ}=17.4 \% ; \quad 135^{\circ}=17.4 \%\right.$; $180^{\circ}=30.5 \%$.

\section{Discussion}

The findings drawn from the image generation task were comparable with the results reported by Kosslyn et al. $(1988,1993)$ with similar tasks. The generation time for complex images was higher than the time required for simple images and the results in the control condition indicated that this could not be exclusively attributed to the perceptual processing of the display (for each of the three groups). This suggested that the generation of mental images of letters took place sequentially. The speed of information processing of the CP-PD group was lower than that of the other groups and this effect was still more pronounced in the image generation condition. The significant interaction between group, complexity and condition also suggested that the rate of sequential activation of visual memory into the visual buffer was slower in these participants. In spite of this slowness of the generation process, the adolescents of the CP-PD group did not make significantly more errors than participants of the two other groups in this image generation task.

The findings of the image maintenance task showed that heavy load items resulted in an increase in memorisation and response times and a decline in performance. The CPPD group again differed from the other groups in terms of its longer response and memorisation times. It also differed from the control group by its lower performance. The significant interaction between group and memory load in memorisation time suggested that the CP-PD group had great difficulty in encoding complex information in the visual buffer. Increasing the complexity of the visual pattern resulted in a sharp increase in encoding time in these participants (from $4264 \mathrm{~ms}$ in light load condition to $9237 \mathrm{~ms}$ in heavy load condition). These difficulties may account for their high error rates in this task.

The increase of response times with distance that was found in the imagery condition of the image scanning task was the hallmark of the scanning process. Response times were higher in the perceptual control condition than in the imagery condition, probably because the display remained visible during the perceptual trials, allowing the participants to take all the time they needed to give their response. Taken together, these results are congruent with the assumption that mental images are scanned using the 
same mechanisms as those that are used in visual perception. Adolescents in the CP-PD group scanned distances at a lower rate than adolescents in the control group in both conditions. The slowness of this process had no effect on the level of performance of these participants but this finding is important for two reasons: firstly, the ease with which one shifts attention over objects or scenes is crucial for a fast adaptation to the environment; and secondly, such a difference has rarely been reported in the literature. Indeed, it seems that the speed of scanning does not vary much across age (Courbois \& Gallina, 1999; Dror \& Kosslyn, 1994) and is not higher in participants with high visuo-spatial abilities (such as pilots, see Dror, Kosslyn, \& Waag, 1993).

In the image rotation task, the linear function relating response time to the angular disparities of the stimuli suggested that an analogous strategy of mental rotation was used. The drop in response times between the orientations $135^{\circ}$ and $180^{\circ}$ for the $\mathrm{S}-$ stimulus may reflect a change in strategy when the stimulus was oriented at $180^{\circ}$. Here again the CP-PD group had slower response times than the $\mathrm{CP}$ and control groups. Participants in the CP-PD group seemed to have difficulty in encoding the $\mathrm{S}-$ stimulus. Reducing the size of the vertical axis resulted in a sharp increase of response times in these participants (from $2895 \mathrm{~ms}$ with the upright $\mathrm{S}+$ stimulus to $6049 \mathrm{~ms}$ with the upright $\mathrm{S}$ - stimulus). Moreover, the speed of the mental rotation process was slower in the CP-PD group than in the two other groups. Despite these characteristics, participants in the CP-PD group did not have higher error rates in mental rotation than participants of the two other groups. These results were congruent with those of Zabadia \& Mellier (1996), who did not find any significant difference in error rates between children with cerebral palsy and control children in a mental rotation task.

The results were congruent with the hypothesis that imagery abilities were poorer for the group with perceptual deficits associated with cerebral palsy than in the two other groups. Response time analysis indicated that the rate of image generation, the speed of scanning and the speed of mental rotation were significantly lower in this group. Moreover, CP-PD participants were also characterised by slower reaction times in all the tasks, even when simple perceptual judgements were required (e.g., the control conditions in the image generation task or the scanning task). This suggested for this group a general slowdown of the processes devoted to visual information encoding, as well as to decision and organisation of the response. Surprisingly, despite this general tendency towards slowness, elementary imagery processes that were analysed in our task appeared to be as efficient in the CP-PD group as in the two other groups. Indeed we did not find significant differences in error rates between the CP-PD group and the others, except in the image maintenance task. Results from this latter task may be related to difficulties in encoding complex patterns, as suggested by the high memorisation times. Although elementary imagery processes seemed to be efficient in these adolescents, the slowness of these processes would probably have a negative impact on performance in more complex spatial problem solving. Indeed, Mumaw, Pellegrino, Kail, \& Carter (1984) have demonstrated that processing time is a crucial factor in accounting for the level of performance in spatial tasks such as those proposed in the Primary Mental Abilities Battery.

However, for most tasks, differences between the CP and the control group were nonsignificant, in considering both response times and error rates (except a trend for the error rates to be higher in the heavy load condition in the CP group). Thus cerebral palsy did not systematically lead to visual imagery impairment. This result is striking since there is a growing literature which suggests that the motor system is intimately involved 
in visual imagery (see Kosslyn, 1994). In image generation tasks for example, it was found that segments of block letters were imaged in the same order that they were drawn (Kosslyn et al., 1988). Furthermore, recent studies argue in favour of common processes for mental object rotation and actual object manipulation (Wexler, Kosslyn, \& Berthoz, 1998; Wohlschläger \& Wohlschläger, 1999). In the present study, participants had a spastic diplegia that primarily affected the lower limbs. They could therefore manipulate the objects without difficulties. This could account for some of the similarities between their results and those of the control group.

The present study constituted a first attempt to clarify the relation between perception and visual mental imagery in persons with cerebral palsy. It seems that spastic diplegic cerebral palsy is not systematically associated with visual imagery deficits. The presence or absence of visual imagery impairment may primarily depend on the existence of perceptual deficits in these persons. Although the lower performance of the CP-PD group with respect to the CP group was obvious, it remains possible that the lack of difference between the $\mathrm{CP}$ and control groups was due to the small sample sizes. Thus the present findings need to be extended to larger samples. They are nevertheless important for the understanding of visual cognition in individuals with cerebral palsy, for two reasons. On the one hand, they suggest that all we know about visual imagery and its development (Dean, 1990; Finke, 1989; Kosslyn, 1994; Richardson, 1999) may be applied to individuals with cerebral palsy without perceptual deficits. On the other hand, they suggest that visual imagery impairments may lead individuals with associated perceptual deficits to specific cognitive profiles. Researchers in cognitive psychology draw a distinction between analogical representations, such as mental images, and propositional representations that are more abstract, language-like representations. Mental images are often used in retrieving information about the visual properties of objects, or about spatial relations among objects, that is not explicitly encoded in an abstract form (Finke, 1989). Such retrieval may be difficult in individuals with cerebral palsy who have associated perceptual deficits. These individuals may try to compensate for their difficulties by relying more on verbal descriptions of visual and spatial information. Indeed, rehabilitation professionals often encourage them to recode visual and spatial information into verbal descriptions. However, this rehabilitation strategy may have some limitations. The visual system implicitly encodes a tremendous amount of information that would be difficult, if not impossible, to encode as verbal descriptions (size, colour, shape, location, orientation of scenes, objects or of their parts). Furthermore, response time analysis in our results suggested that individuals in the CP-PD group were able to implement visual imagery processes, albeit at a slower rate. Given the importance of mental imagery in retrieving information from memory or in representing spatial transformations, it may be important to add some imagery training programs to the rehabilitation programs designed for these individuals.

\section{Acknowledgement}

The authors are grateful to Emilie Delattre for her help in collecting data. This research was supported by the Institut Régional de Recherche sur le Handicap. 


\section{References}

Black, P. D. (1980). Ocular defects in children with cerebral palsy. British Medical Journal, 281, 487-488.

Breakley, A. S., Wilson, J. J., \& Wilson, B. C. (1974). Sensory and perceptual functions in the cerebral palsied. Journal of Nervous and Mental Disease, 158(1), 70-77.

Courbois, Y. (2000). The role of stimulus axis salience in children's ability to mentally rotate unfamiliar figures. European Journal of Cognitive Psychology, 12(2), 261-269.

Courbois, Y., \& Gallina, J. M. (1999). Scanning visual mental images: A developmental point of view. Current Psychology of Cognition, 18(4), 502-508.

Dean, A. L. (1990). The development of mental imagery: A comparison of piagetian and cognitive psychological perspectives. Annals of Child Development, 7, 105-144.

Denis, M. (1991). Image and Cognition. New York: Harvester Wheatsheaf.

Denis, M., \& Kosslyn, S. M. (1999). Scanning visual mental images: A window on the mind. Current Psychology of Cognition, 18(4), 409-465.

Dror, I. E., \& Kosslyn, S. M. (1994). Mental imagery and aging. Psychology and Aging, 9, 90-102.

Dror, I. E., Kosslyn, S. M., \& Waag, W. L. (1993). Visuo-spatial abilities of pilots. Journal of Applied Psychology, 78(5), 763-773.

Finke, R. A. (1989). Principles of mental imagery. Cambridge, MA: MIT Press.

Finke, R. A., \& Pinker, S. (1982). Spontaneous imagery scanning in mental extrapolation. Journal of Experimental Psychology: Learning, Memory, and Cognition, 8, 142-147.

Foreman, N., Orencas, C., Nicholas, E., Morton, P., \& Gell (1989). Spatial awareness in seven to 11-year-old physically handicapped children in mainstream schools. European Journal of Special Needs Education, 4, 171-179.

Kosslyn, S. M. (1980). Image and mind. Cambridge: Harvard University Press.

Kosslyn, S. M. (1994). Image and brain: The resolution of the imagery debate. Cambridge, MA: MIT Press.

Kosslyn, S. M., Alpert, N. M., Thompson, W. L., Maljkovic, V., Weise, S. B., Chabris, C. F., Hamilton, S. E., Rauch, S. L., \& Buonanno, F. S. (1993). Visual mental imagery activates topographically organized visual cortex: PET investigations. Journal of Cognitive Neuroscience, 5, 263-287.

Kosslyn, S. M., Cave, B. S., Provost, D. A., \& Von Gierke, S. M. (1988). Sequential processes in image generation. Cognitive Psychology, 20, 319-343.

Kosslyn, S. M., Margolis, J. A., Barrett, A. M., Goldknopf, E. J., \& Daly, P. F. (1990). Age differences in imagery abilities. Child Development, 61, 995-1010.

Kosslyn, S. M., Thompson, W. L., Kim, I. J., \& Alpert, N. M. (1995). Topographical representations of mental images in primary visual cortex. Nature, 378, 496-498.

Mazeau, M. (1989). Troubles du regard et échec scolaire chez l'IMC; conséquences scolaires de l'apraxie visuo-spatiale. Motricité Cérébrale, 10(1), 1-16.

Mumaw, R. J., Pellegrino, J., Kail, R., \& Carter, P. (1984). Different slopes for different folks: Process analysis of spatial aptitude. Memory and Cognition, 12, 515-521.

Richardson, J. T. E. (1999). Imagery. Hove: Psychology Press.

Shepard, R. N., \& Metzler, J. (1971). Mental rotation of three-dimensional objects. Science, 171, 701-703.

Wexler, M., Kosslyn, S. M., \& Berthoz, A. (1998). Motor processes in mental rotation. Cognition, $68,77-94$.

Wohlschläger, A., \& Wohlschläger, A. (1999). Mental and manual rotation. Journal of Experimental Psychology: Human Perception and Performance, 24(2), 397-412.

Zabadia, M., \& Mellier, D. (1996). Effet de l'exercice de rotation mentale sur le traitement des relations topologiques élémentaires chez des enfants handicapés moteurs. Archives de Psychologie, 64, 67-82. 